\title{
Diagnostic use of nuclear $\beta$-catenin expression for the assessment of endometrial stromal tumors
}

\author{
Chan-Kwon Jung ${ }^{1}$, Ji-Han Jung ${ }^{1}$, Ahwon Lee ${ }^{1}$, Youn-Soo Lee ${ }^{1}$, Yeong-Jin Choi ${ }^{1}$, \\ Seung-Kew Yoon ${ }^{2}$ and Kyo-Young Lee ${ }^{1}$ \\ ${ }^{1}$ Department of Hospital Pathology, College of Medicine, The Catholic University of Korea, Seoul, Republic \\ of Korea and ${ }^{2}$ Department of Internal Medicine, College of Medicine, The Catholic University of Korea, Seoul, \\ Republic of Korea
}

\begin{abstract}
Alterations in $\beta$-catenin degradation cause it to accumulate to immunohistochemically detectable levels in the nuclei of tumor cells. Although it has been shown that nuclear $\beta$-catenin immunostaining is useful for the diagnosis of some mesenchymal tumors, there is little known about $\beta$-catenin expression in endometrial stromal tumors. In this study, nuclear $\beta$-catenin immunoreactivity was evaluated in normal endometrium and endometrial mesenchymal tumors and then compared with that of CD10. The endometrial mesenchymal tumors evaluated included endometrial stromal nodules $(n=2)$, low-grade endometrial stromal sarcomas $(n=12)$, undifferentiated endometrial sarcomas $(n=8)$ and uterine cellular leiomyomata $(n=9)$. In addition, direct DNA sequencing of $\beta$-catenin exon 3 was conducted in 15 endometrial stromal tumors. Normal endometrial stromal cells showed strong cytoplasmic reactivity for CD10 but no detectable reactivity for $\beta$-catenin. Nuclear $\beta$-catenin immunoreactivity was detected in 11 low-grade endometrial stromal sarcomas (92\%) and 6 undifferentiated endometrial sarcomas $(75 \%)$. Ten low-grade endometrial stromal sarcomas $(83 \%)$ and six undifferentiated endometrial sarcomas $(75 \%)$ were positive for CD10. Eight low-grade endometrial stromal sarcomas (67\%) exhibited diffuse, strong nuclear immunoreactivity with $\beta$-catenin, whereas only four cases $(33 \%)$ expressed diffuse, strong immunoreactivity with CD10. All nine cases of uterine cellular leiomyomata were completely negative for both $\mathrm{CD} 10$ and $\beta$-catenin. $\beta$-catenin mutations were rare in endometrial stromal tumors. Taken together, these results indicate that nuclear $\beta$-catenin immunostaining can serve as a sensitive immunohistochemical marker for the diagnosis of endometrial stromal tumors and is useful for differentiating low-grade endometrial stromal sarcomas from uterine cellular leiomyomata.
\end{abstract}

Modern Pathology (2008) 21, 756-763; doi:10.1038/modpathol.2008.53; published online 28 March 2008

Keywords: endometrial stromal tumor; $\beta$-catenin; CD10; immunohistochemistry

It is well known that $\beta$-catenin plays a dual role in cell adhesion and transcriptional activation. ${ }^{1} \beta$ catenin binds to cadherin adhesion receptors, and associates with the actin cytoskeleton via $\alpha$-catenin to form cell-cell adherens junctions. Disruption of this cell-cell adhesion in malignant transformed cells may contribute to the enhanced migration and proliferation of tumor cells, leading to invasion and metastasis. In addition to its structural role in intercellular adherens junctions, $\beta$-catenin can also act as a transcription factor in the nucleus by serving

Correspondence: Dr K-Y Lee, MD, PhD., Department of Hospital Pathology, College of Medicine, The Catholic University of Korea, 505, Banpo-dong, Seocho-gu, Seoul 137-701, Republic of Korea. E-mail: leekyoyo@catholic.ac.kr

Received 03 January 2008; revised 13 February 2008; accepted 14 February 2008; published online 28 March 2008 as a co-activator of the lymphoid enhancer factor (LEF)/T-cell factor (TCF) family of DNA-binding proteins. $\beta$-catenin-mediated transcription is activated by the Wnt signaling pathway, which also plays a role in tumorigenesis. Activation of Wnt signaling involves the inhibition of $\beta$-catenin degradation by proteasomes, which leads to nuclear accumulation and transcriptional activation of $\mathrm{LEF} / \mathrm{TCF}$ target genes. Mutations in components that regulate $\beta$-catenin turnover (eg, APC, AXIN or GSK3B), as well as mutations in $\beta$-catenin itself that compromise the protein's degradation, have been found in a variety of human cancers. ${ }^{2}$ These mutations lead to an excessive accumulation of $\beta$ catenin in the nucleus and stimulation of its target genes in an aberrant manner, which subsequently results in neoplasia. ${ }^{2}$ This nuclear accumulation can result in an adequately high concentration of 
$\beta$-catenin in the nuclei of tumor cells to allow it to be detected immunohistochemically. ${ }^{3}$

Nuclear accumulation of $\beta$-catenin has been observed in some types of mesenchymal tumors, including desmoid-type fibromatosis, solitary fibrous tumor, synovial sarcoma, osteosarcoma, liposarcoma, malignant fibrous histiocytoma and mesenteric fibromatosis. ${ }^{3-7}$ Although nuclear accumulation of $\beta$-catenin has recently been demonstrated in endometrial stromal sarcomas, these studies included only a small sample size and immunostaining produced variable results. ${ }^{3,8}$

It has been reported that CD10 is expressed by normal and neoplastic endometrial stromal cells, as well as stromal cells involved in endometriosis and adenomyosis, ${ }^{9-11}$ and diffuse CD10 immunoreactivity is a very useful positive predictive marker for endometrial stromal sarcomas. ${ }^{9}$ However, a study conducted by McCluggage et $a 1^{10}$ showed that $38 \%$ of low-grade endometrial stromal sarcomas expressed focally weak CD10 positivity.

The purpose of the present study was to compare the usefulness of $\beta$-catenin and CD10 immunostaining for the characterization of endometrial stromal tumors, and to determine the effectiveness of these markers for distinguishing endometrial stromal tumors from uterine cellular leiomyomata in paraffin-embedded tissue sections. To evaluate whether $\beta$-catenin mutations are present in endometrial stromal tumors, we also sequenced exon 3 of the $\beta$-catenin gene, which contains GSK3B phosphorylation sites.

\section{Materials and methods}

\section{Case Selection}

Cases were obtained from the files of the Department of Hospital Pathology, Catholic Medical Center, The Catholic University of Korea. The selected cases consisted of 15 normal endometrial tissue samples (5 proliferative, 5 secretory and 5 atrophic), 2 endometrial stromal nodules, 12 low-grade endometrial stromal sarcomas, 8 undifferentiated endometrial sarcomas and 9 uterine cellular leiomyomata. Determination of the tumor type and histopathologic grading was performed according to the new World Health Organization classification on Tumors of the Breast and Female Genital Organs. ${ }^{12}$ A representative section from each case containing the tumor was chosen for the study.

\section{Immunohistochemistry}

Formalin-fixed, paraffin-embedded tissue blocks were cut into 4- $\mu$ m-thick serial sections and mounted on silanized glass slides. The tissue sections were then deparaffinized, rehydrated and rinsed in distilled water. Next, immunohistochemical assays for $\beta$-catenin and CD10 were performed on consecutive paraffin sections using an automated immunohistochemical stainer (Lab Vision Autostainer LV-1; LabVision/Neomarkers, Fremont, CA, USA). Antigen retrieval was then performed using $0.01 \mathrm{M}$ citrate buffer ( $\mathrm{pH} 6.0$ ) by heating the sample in a microwave vacuum histoprocessor (RHS-1, Milestone, Bergamo, Italy) at a controlled final temperature of $121^{\circ} \mathrm{C}$ for $15 \mathrm{~min}$. The endogenous peroxidase activity was then blocked by incubating the slides in $3 \%$ hydrogen peroxide in methanol for $10 \mathrm{~min}$. The primary antibodies were diluted in Dako Antibody Diluent (Dako, Carpinteria, CA, USA) with background-reducing components and then used at the following dilutions: $\beta$-catenin (1:200, clone 14, monoclonal, Becton Dickinson Transduction Labs, San Diego, CA, USA) and CD10 (1:400, clone 56C6, monoclonal, Novocastra Labs, Newcastle upon Tyne, UK). Next, the primary antibodies were incubated at room temperature for $30 \mathrm{~min}$, after which they were detected using the Envision plus System (Dako). The immunoreaction was developed with diaminobenzidine (DAB; Dako) for $5 \mathrm{~min}$, and then counterstained with hematoxylin.

We considered only the nuclear staining pattern of $\beta$-catenin and cytoplasmic staining of CD10 as positive. Positive controls for $\beta$-catenin consisted of a fibromatosis obtained from a patient with a known $A P C$ gene alteration. In addition, normal endometrial stromal tissue was used as an internal positive control for CD10. For negative controls, phosphate buffered saline was used instead of the primary antibody.

The degree of immunoreactivity for $\beta$-catenin and CD10 was evaluated semi-quantitatively on the basis of staining intensity and the percentage of positive cells, as described previously. ${ }^{10}$ The staining intensity was classified as negative, weak, moderate or strong expression. The staining percentage was classified as focal or diffuse using $50 \%$ as a cutoff point.

\section{DNA Extraction}

DNA was extracted from two $10-\mu \mathrm{m}$ thick paraffin sections containing a representative portion of each tumor block using the QIAamp DNA Mini kit (Qiagen, Hilden, Germany). Neoplastic areas were manually microdissected from the tissue sections.

\section{Polymerase Chain Reaction and Sequencing of $\beta$-Catenin}

A $217 \mathrm{bp}$ fragment of exon 3 of the $\beta$-catenin gene was amplified using sense primer ( $5^{\prime}$-ATTTGATGG AGTTGGACATGG- $\left.3^{\prime}\right)$ and antisense primer $\left(5^{\prime}\right.$-TG TTCTTGAGTGAAGGACTGA-3') in a $20 \mu$ l reaction solution containing $10 \mu \mathrm{l}$ of $2 \times$ HotStarTaq Master Mix (Qiagen), as described previously. ${ }^{13}$ Polymerase chain reaction (PCR) condition was as follows: 30 
cycles of $30 \mathrm{~s}$ at $94^{\circ} \mathrm{C}, 1 \mathrm{~min}$ at $55^{\circ} \mathrm{C}$ and $1 \mathrm{~min}$ at $72^{\circ} \mathrm{C}$. Water was used instead of template DNA for a negative control. PCR products were then evaluated by $2 \%$ agarose gel electrophoresis, after which they were extracted from the gels and purified using a HiYield ${ }^{\mathrm{TM}} \mathrm{Gel} / \mathrm{PCR}$ DNA Extraction Kit (RBC bioscience, Taipei, Taiwan). Purified PCR products were processed for the DNA sequencing reaction using the ABI-PRISM BigDye Terminator version 3.1 (Applied Biosystems, Foster, CA, USA), according to the manufacturer's protocols. Sequencing was performed in both directions using the same primers used for PCR. Sequencing reactions were performed using 25 cycles of $10 \mathrm{~s}$ at $96^{\circ} \mathrm{C}, 5 \mathrm{~s}$ at $50^{\circ} \mathrm{C}$ and $4 \mathrm{~min}$ at $60^{\circ} \mathrm{C}$. Sequence data were generated using the ABI PRISM 3100 DNA Analyzer (Applied Biosystems) and the sequences were then analyzed and compared using the Sequencer 3.1.1. software (Applied Biosystems).

\section{Results}

The results for the $\beta$-catenin and CD10 immunohistochemistry are illustrated in the Figure 1.

\section{Immunoreactivity of $\beta$-Catenin and CD10 in Normal Endometrium}

The epithelial cells obtained from all cases showed strong membranous reactivity for $\beta$-catenin without nuclear staining, but no reactivity for CD10. Stromal cells showed strong cytoplasmic reactivity for CD10 but no detectable reactivity for $\beta$-catenin (Figure 2).

\section{Immunoreactivity of $\beta$-Catenin and CD10 in Endometrial Stromal Tumors}

Nuclear reactivity for $\beta$-catenin was noted in 2 $(100 \%)$ of the 2 cases of endometrial stromal nodules evaluated, 11 (92\%) of the 12 low-grade endometrial stromal sarcoma cases evaluated and 6 $(75 \%)$ of the 8 undifferentiated endometrial sarcomas evaluated. Cytoplasmic reactivity for CD10 was noted in $2(100 \%)$ of the 2 cases of endometrial stromal nodules evaluated, $10(83 \%)$ of the 12 lowgrade endometrial stromal sarcomas evaluated and 6 $(75 \%)$ of the 8 undifferentiated endometrial sarcomas evaluated. The staining pattern of these antibodies was generally diffuse and strong in low-grade endometrial stromal sarcomas (Figure 3). However, there were some differences in the staining pattern that occurred as a result of CD10 and $\beta$-catenin immunoreactivity (Figure 4). Eight cases of low-grade endometrial stromal sarcomas (67\%) exhibited diffuse, strong nuclear immunoreactivity when $\beta$-catenin was evaluated, whereas only four cases $(33 \%)$ expressed diffuse, strong immunoreactivity when CD10 was evaluated. In addition, two cases of low-grade endometrial stromal sarcomas

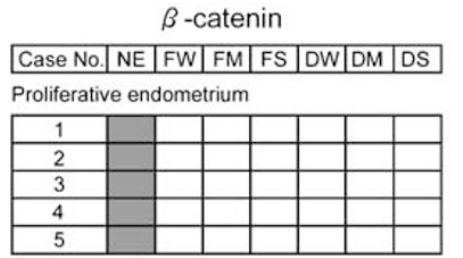

Secretory endometrium

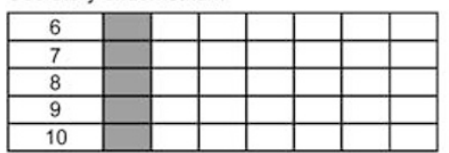

Atrophic endometrium

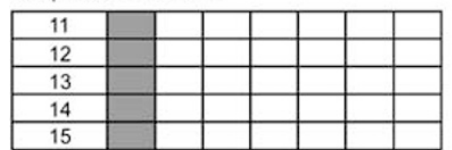

\begin{tabular}{|c|c|c|c|c|}
\hline 15 & & & & \\
\hline Endometrial stromal nodule
\end{tabular}

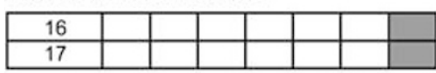

Endometrial stromal sarcoma, low grade

\begin{tabular}{|l|l|l|l|l|l|l|l|}
\hline 18 & & & & & & & \\
\hline 19 & & & & & & & \\
\hline 20 & & & & & & & \\
\hline 21 & & & & & & & \\
\hline 22 & & & & & & & \\
\hline 23 & & & & & & & \\
\hline 24 & & & & & & & \\
\hline 25 & & & & & & & \\
\hline 26 & & & & & & & \\
\hline 27 & & & & & & & \\
\hline 28 & & & & & & & \\
\hline 29 & & & & & & & \\
\hline
\end{tabular}

Undifferentiated endometrial sarcoma

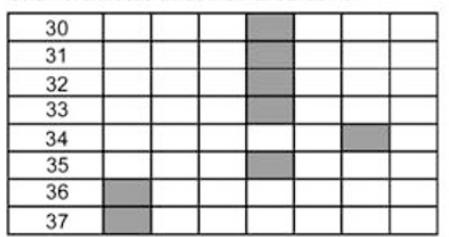

Cellular leiomyoma
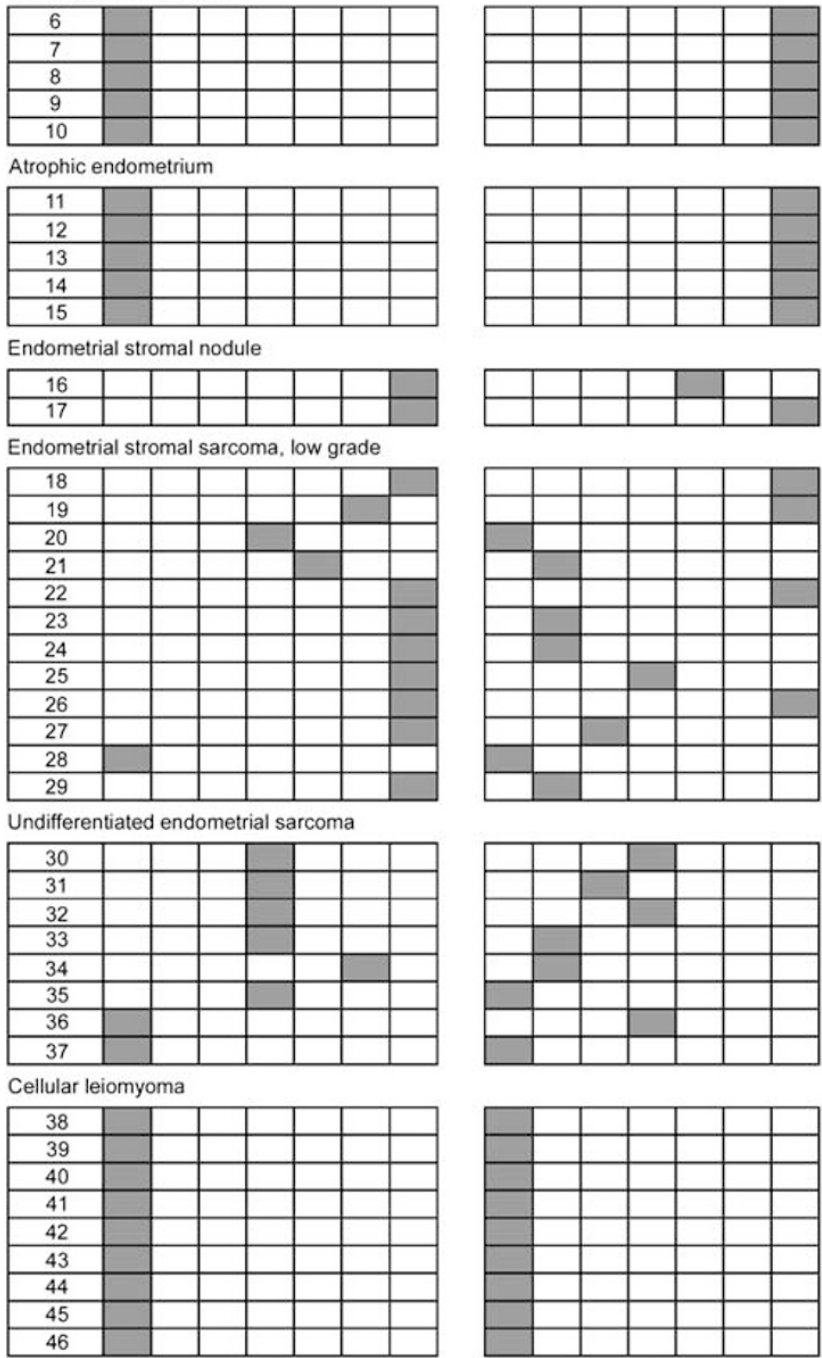

Figure 1 Illustration demonstrating the results of the immunohistochemical analysis. DM, diffuse moderate; DS, diffuse strong; DW, diffuse weak; FM, focal moderate; FS, focal strong; FW, focal weak; NE, negative.

were completely negative for CD10, whereas one (case 20) of these cases showed strong focal staining for $\beta$-catenin, and the other (case 28) was negative for $\beta$-catenin. Immunoreactivity for both antibodies in undifferentiated endometrial sarcomas usually showed a focal staining pattern, and the positive rates of both of these antibodies was also the same. However, the staining intensity of $\beta$-catenin was intense in most cases of undifferentiated endometrial sarcomas, whereas the intensity of the CD10 staining varied from weak to strong. 

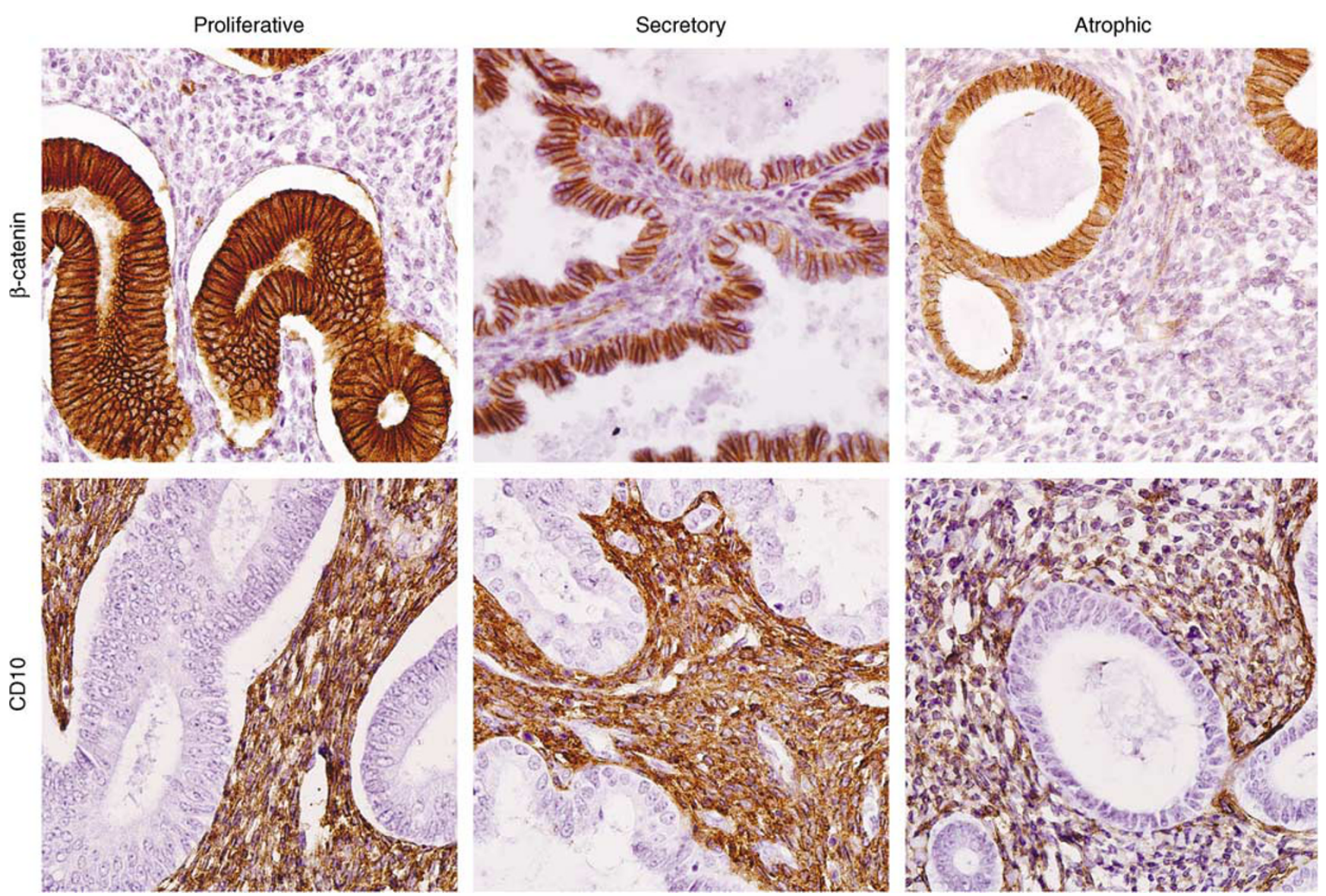

Figure 2 Immunoreactivity for $\beta$-catenin and CD10 in proliferative, secretory and atrophic endometrium. The endometrial glands show membranous positivity for $\beta$-catenin without nuclear staining, whereas the endometrial stroma are negative. Conversely, reactivity for CD10 is limited to stromal cells. No CD10 staining was observed in glands.

\section{Immunoreactivity for $\beta$-Catenin and CD10 in Uterine Cellular Leiomyomata}

All nine cases of uterine cellular leiomyomata were negative for both $\beta$-catenin and CD10 antibodies.

\section{DNA Sequencing of Exon 3 of the $\beta$-Catenin Gene}

To assess the prevalence of $\beta$-catenin mutations in endometrial stromal tumors, as well as to determine whether there was a strong correlation between nuclear $\beta$-catenin staining and $\beta$-catenin mutations, we conducted direct sequencing of the PCR products generated from genomic DNA preparations of the specimens.

Sufficient DNA was recovered from 15 of the specimens (2 endometrial stromal nodules, 7 low-grade endometrial stromal sarcomas and 6 undifferentiated endometrial sarcomas), however, only 1 of these specimens (case 16, endometrial stromal nodules) was found to have a $\beta$-catenin mutation. The mutation, which was a TCT to AAT transition at codon 33 that resulted in a change from serine to asparagine, was confirmed in independent PCR and sequencing reactions (Figure 5).

\section{Discussion}

Endometrial stromal sarcomas are rare uterine mesenchymal tumors that have traditionally been divided into low and high grades based on mitotic count. The recent World Health Organization Classification of Tumors of the Breast and Female Genital Organs ${ }^{12}$ divides endometrial mesenchymal tumors into three groups: (1) benign endometrial stromal nodules, (2) endometrial stromal sarcomas, low-grade and (3) undifferentiated endometrial sarcomas. The distinction between benign and malignant groups is made based on the type of tumor margin. Malignant tumors are subdivided into low-grade endometrial stromal sarcomas and undifferentiated endometrial sarcomas based on features such as nuclear pleomorphism and necrosis.

Undifferentiated endometrial sarcomas are aggressive, with death usually occurring within 3 years of hysterectomy, whereas low-grade endometrial stromal sarcomas are indolent tumors with late recurrences and distant metastasis. ${ }^{12}$ When presenting as extrauterine or metastatic disease, these tumors may be difficult to differentiate from other soft-tissue 

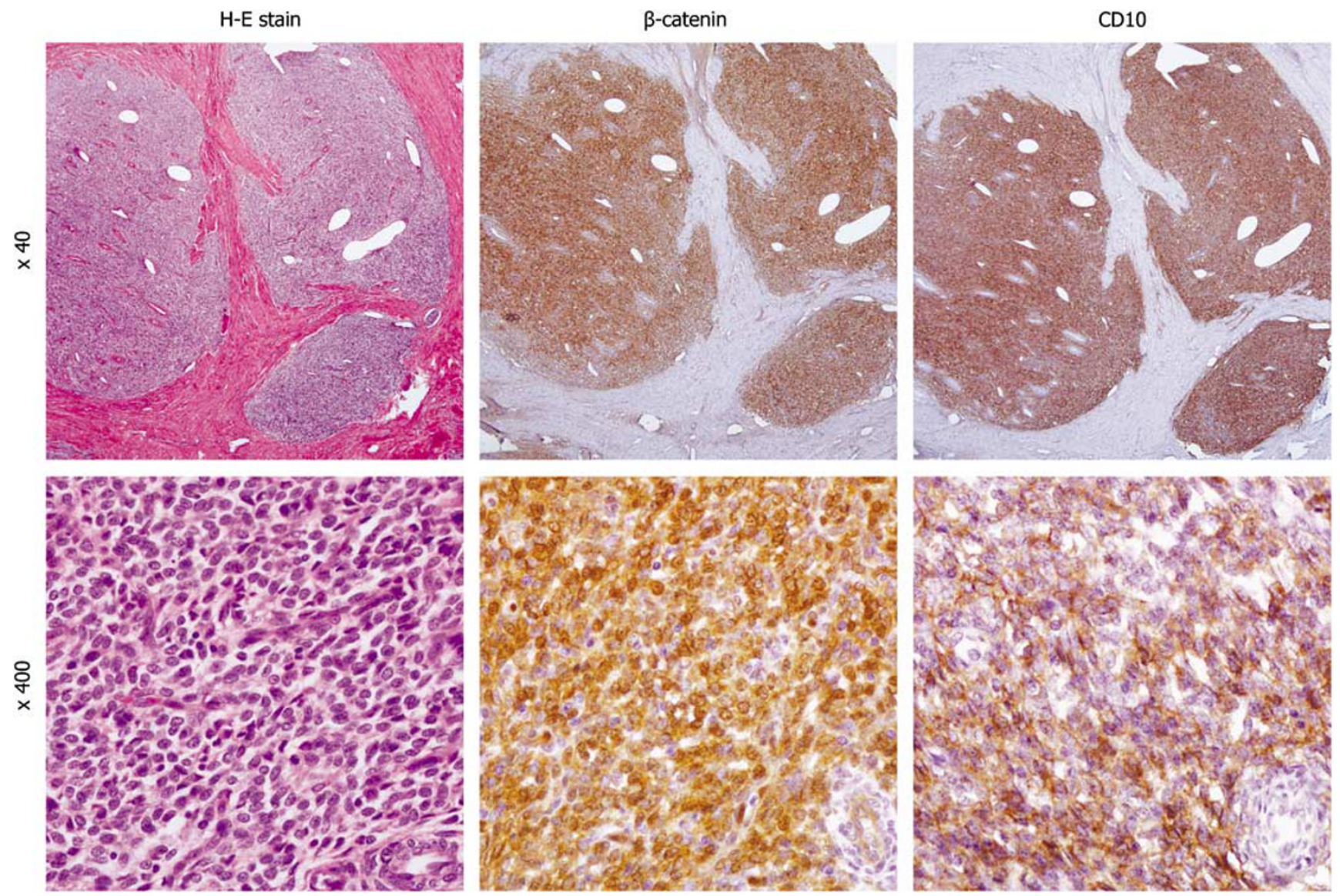

Figure 3 Low-grade endometrial stromal sarcoma (case 22$)$ at low power $(\times 40)$ and high power $(\times 400)$ views. Tumor cells show diffuse and strong immunoreactivity for $\beta$-catenin and CD10. $\beta$-catenin immunostaining in a low-grade endometrial stromal sarcoma shows nuclear and cytoplasmic staining of tumor cells and cytoplasmic staining of endothelial cells.

tumors, especially in the absence of information concerning past medical history. However, immunohistochemistry may be useful for diagnosis in such cases. Immunohistochemical studies of endometrial stromal sarcomas have shown that they are usually positive for CD10 and both estrogen and progesterone receptors, and that they may also be positive for smooth muscle markers (such as actin and desmin). ${ }^{10-12,14}$ Although diffuse strong immunoreactivity for CD10 favors the diagnosis of endometrial stromal sarcomas, ${ }^{9}$ it is important to note that this diagnosis is not always straightforward. For example, the staining may be focal or weak, which could result in a false negative. In this study, we investigated CD10 expression in 12 cases of low-grade endometrial stromal sarcomas and 8 cases of undifferentiated endometrial sarcomas. Six $(50 \%)$ of the low-grade endometrial stromal sarcomas and $6(75 \%)$ of the undifferentiated endometrial sarcomas showed focal staining for CD10, whereas only $4(33 \%)$ of the low-grade endometrial stromal sarcomas and none of the undifferentiated endometrial sarcomas were diffusely positive for CD10. Because hormone receptors usually stain uterine smooth muscle tumors, these markers have little reliability in differentiating endometrial stromal tumors from uterine smooth muscle tumors. ${ }^{9,15,16}$ Although CD10 is a useful immunohistochemical marker for the differential diagnosis of endometrial stromal sarcomas from uterine cellular leiomyomata, CD10 may also be detected in uterine cellular leiomyomata. ${ }^{9,11}$

In this study, we demonstrated that $\beta$-catenin nuclear expression is frequently found in endometrial stromal tumors, but not in normal endometrial stroma and uterine cellular leiomyomata. Nuclear reactivity for $\beta$-catenin was noted in both of the 2 endometrial stromal nodules evaluated in this study, in $11(92 \%)$ of the 12 low-grade endometrial stromal sarcomas evaluated and in $6(75 \%)$ of the 8 undifferentiated endometrial sarcomas evaluated. Among three cases of CD10-negative endometrial stromal tumors, two (case 20 of endometrial stromal sarcomas and case 35 of undifferentiated endometrial sarcomas) were positive for $\beta$-catenin. When the low-grade endometrial stromal sarcomas were evaluated there were some differences observed in the staining pattern and intensity of CD10 and $\beta$-catenin immunoreactivity, with eight cases of low-grade endometrial stromal sarcomas (67\%) exhibiting 

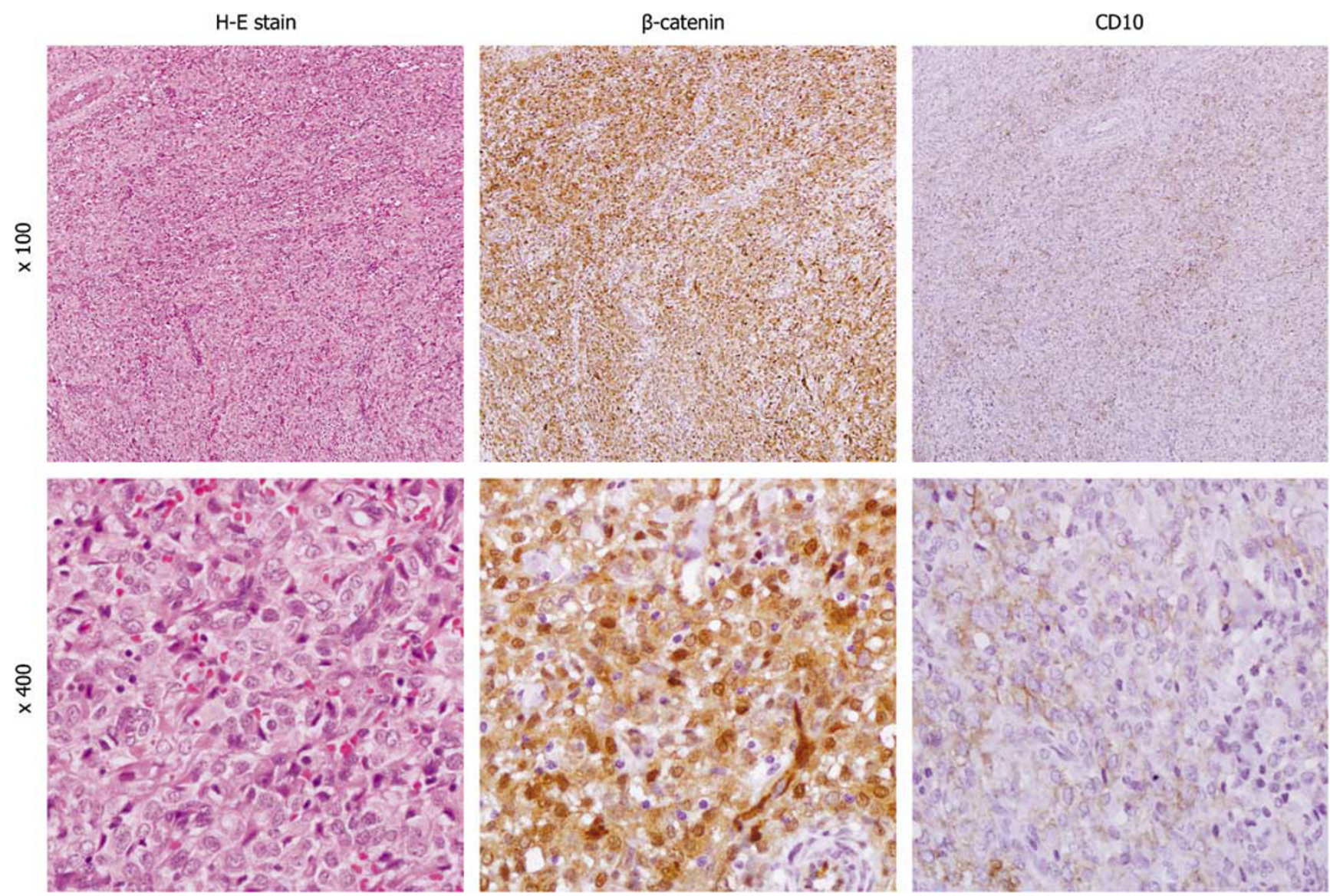

Figure 4 In low-grade endometrial stromal sarcoma (case 29), tumor cells show diffuse and strong immunoreactivity for $\beta$-catenin, whereas these tumor cells are focally and weakly positive for CD10. $\beta$-catenin immunostaining in a low-grade endometrial stromal sarcoma shows nuclear and cytoplasmic staining of tumor cells and cytoplasmic staining of endothelial cells.
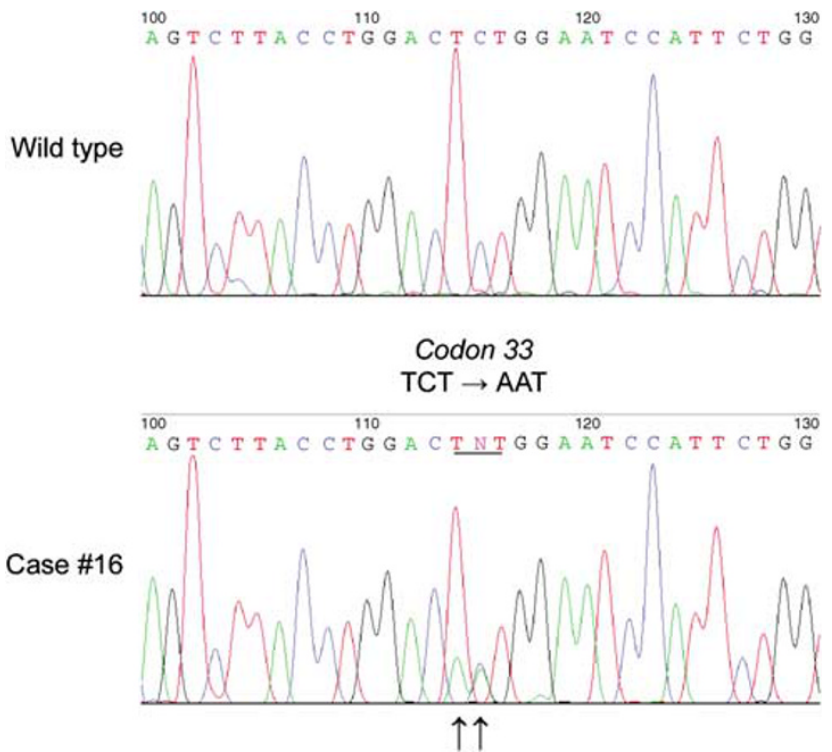

Figure 5 Representative electropherograms of sequence analysis of exon 3 of the $\beta$-catenin gene in endometrial stromal tumors. The mutation of TC to AA at codon 35, which results in a change from serine to asparagine, is indicated by an arrow. diffuse, strong nuclear immunoreactivity with $\beta$ catenin, and only four cases (33\%) showing diffuse, strong immunoreactivity with CD10. Therefore, such focal staining for CD10 will make the exclusion of endometrial stromal tumors difficult in small biopsy specimens. Based on these results, inclusion of $\beta$-catenin in a panel of immunohistochemical markers may be useful for confirming a diagnosis of endometrial stromal tumors and differentiating low-grade endometrial stromal sarcomas from uterine cellular leiomyomata.

Correct interpretation of positive $\beta$-catenin staining is important. Only nuclear staining is considered significant when separating endometrial stromal tumors from other entities in the differential diagnosis. In our study, cytoplasmic staining was seen in uterine cellular leiomyomata as well as in endometrial stromal tumors.

To the best of our knowledge only two studies regarding $\beta$-catenin immunohistochemistry in endometrial stromal sarcomas can be found in the English literature. ${ }^{3,8}$ The anti- $\beta$-catenin monoclonal antibody used in our study was the same as that used in a recent study conducted by $\mathrm{Ng}$ et $a{ }^{3},{ }^{3}$ in 
which the authors examined the nuclear expression of $\beta$-catenin by immunohistochemical staining of tissue microarrays and found that a high-level ( $>25 \%$ of cells) of staining occurred in $2(40 \%)$ of 10 endometrial stromal sarcomas evaluated, but that a low-level (0-25\% of cells) of staining also occurred in $2(20 \%)$ of the cases. This finding is different from the results of the present study, in which diffuse staining of $\beta$-catenin was found in 8 $(67 \%)$ of 12 low-grade endometrial stromal sarcomas and focal staining was observed in only 1 low-grade endometrial stromal sarcoma. This difference in results may be the consequence of case selection or the use of whole tissue sections vs tissue microarray sections. Interestingly, another study showed different staining patterns from those of $\mathrm{Ng}$ et $\mathrm{al}^{3}$ and our results. Hrzenjak et $a l^{8}$ found that $\beta$-catenin was only weakly positive in the cytoplasm of tumor cells and that tumor cell nuclei were mostly negative in 10 endometrial stromal sarcomas, whereas tumor cells from 4 cases of undifferentiated endometrial sarcomas showed nuclear expression of $\beta$-catenin. Although it is unclear as to why these differences occurred, the use of a different commercial antibodies for $\beta$-catenin may have produced different immunoreactivity patterns. Hrzenjak et $a l^{8}$ used a monoclonal $\beta$-catenin antibody purchased from Dako (Copenhagen, Denmark), whereas a BD Transduction Laboratories anti- $\beta$-catenin monoclonal antibody was used in this study and the study conducted by $\mathrm{Ng}$ et $a l^{3}$.

$\beta$-catenin mutations have been identified in a wide variety of human cancers at low frequencies; for example, $10-15 \%$ in colon carcinomas, ${ }^{17,18} 13 \%$ in endometrial carcinomas, ${ }^{19} 8 \%$ in ovarian carcinomas, ${ }^{20} 7.5 \%$ in biliary tract carcinomas, ${ }^{21} 5 \%$ in prostate carcinomas ${ }^{22}$ and $4 \%$ in medulloblastomas. ${ }^{23}$ However, $\beta$-catenin mutations are far more common in certain histological subtypes; for example, $75 \%$ in pilomatricoma of the skin, ${ }^{24} 61 \%$ in anaplastic thyroid carcinoma ${ }^{25}$ and $52 \%$ in sporadic aggressive fibromatoses. ${ }^{26}$ Most of the previously reported $\beta$-catenin point mutation sites in other human benign and malignant tumors were at codons 32 (Asp), 33 (Ser), 34 (Gly), 37 (Ser), 41 (Thr) and 45 (Ser). ${ }^{2}$ In our study, we attempted to find genetic alterations of the $\beta$-catenin gene in 15 endometrial stromal tumors, but only one of these cases (case 16, endometrial stromal nodules) harbored a $\beta$-catenin mutation at codon 33 (TCT to AAT, Ser to Asn). This indicates that nuclear expression of $\beta$-catenin was frequently observed in endometrial stromal tumors, but that $\beta$-catenin mutations were rare events. Furthermore, because nuclear accumulation of $\beta$-catenin is a potential indicator of the activation of the Wnt signaling pathway, the mechanism by which $\beta$-catenin accumulation in endometrial stromal tumors without mutation occurs needs to be further elucidated.

In summary, our study showed that nuclear $\beta$-catenin was not expressed in normal endometrial stroma or uterine smooth muscle tumors, but that it was frequently expressed in endometrial stromal tumors, although its expression is decreased in undifferentiated endometrial sarcomas. These characteristics indicate that $\beta$-catenin can serve as a sensitive and useful immunohistochemical marker for the diagnosis of endometrial stromal tumors. $\beta$-catenin staining can also complement the use of CD10 in the differential diagnosis of these tumors.

\section{Conflict of interest}

None to declare.

\section{References}

1 Conacci-Sorrell M, Zhurinsky J, Ben-Ze'ev A. The cadherin-catenin adhesion system in signaling and cancer. J Clin Pathol 2002;109:987-991.

2 Polakis P. Wnt signaling and cancer. Genes Dev 2000; 14:1837-1851.

3 Ng TL, Gown AM, Barry TS, et al. Nuclear beta-catenin in mesenchymal tumors. Mod Pathol 2005;18: $68-74$.

4 Haydon RC, Deyrup A, Ishikawa A, et al. Cytoplasmic and/or nuclear accumulation of the beta-catenin protein is a frequent event in human osteosarcoma. Int J Cancer 2002;102:338-342.

5 Sakamoto A, Oda Y, Adachi T, et al. Beta-catenin accumulation and gene mutation in exon 3 in dedifferentiated liposarcoma and malignant fibrous histiocytoma. Arch Pathol Lab Med 2002;126: 1071-1078.

6 Bhattacharya B, Dilworth HP, Iacobuzio-Donahue C, et al. Nuclear beta-catenin expression distinguishes deep fibromatosis from other benign and malignant fibroblastic and myofibroblastic lesions. Am J Surg Pathol 2005;29:653-659.

7 Montgomery E, Torbenson MS, Kaushal M, et al. Betacatenin immunohistochemistry separates mesenteric fibromatosis from gastrointestinal stromal tumor and sclerosing mesenteritis. Am J Surg Pathol 2002;26: 1296-1301.

8 Hrzenjak A, Tippl M, Kremser ML, et al. Inverse correlation of secreted frizzled-related protein 4 and beta-catenin expression in endometrial stromal sarcomas. J Pathol 2004;204:19-27.

9 Chu PG, Arber DA, Weiss LM, et al. Utility of CD10 in distinguishing between endometrial stromal sarcoma and uterine smooth muscle tumors: an immunohistochemical comparison of 34 cases. Mod Pathol 2001;14: 465-471.

10 McCluggage WG, Sumathi VP, Maxwell P. CD10 is a sensitive and diagnostically useful immunohistochemical marker of normal endometrial stroma and of endometrial stromal neoplasms. Histopathology 2001; 39:273-278.

11 Toki T, Shimizu M, Takagi Y, et al. CD10 is a marker for normal and neoplastic endometrial stromal cells. Int J Gynecol Pathol 2002;21:41-47.

12 Tavassoli FA, Devilee P. World Health Organization Classification of Tumors: Pathology and Genetics of Tumors of the Breast and Female Genital Organs. IARC Press: Lyon, France, 2003. 
13 Saegusa M, Okayasu I. Frequent nuclear beta-catenin accumulation and associated mutations in endometrioid-type endometrial and ovarian carcinomas with squamous differentiation. J Pathol 2001;194:59-67.

14 Franquemont DW, Frierson Jr HF, Mills SE. An immunohistochemical study of normal endometrial stroma and endometrial stromal neoplasms. Evidence for smooth muscle differentiation. Am J Surg Pathol 1991;15:861-870.

15 Sutton GP, Stehman FB, Michael H, et al. Estrogen and progesterone receptors in uterine sarcomas. Obstet Gynecol 1986;68:709-714.

16 Navarro D, Cabrera JJ, Leon L, et al. Endometrial stromal sarcoma expression of estrogen receptors, progesterone receptors and estrogen-induced srp27 (24 K) suggests hormone responsiveness. J Steroid Biochem Mol Biol 1992;41:589-596.

17 Samowitz WS, Powers MD, Spirio LN, et al. Beta-catenin mutations are more frequent in small colorectal adenomas than in larger adenomas and invasive carcinomas. Cancer Res 1999;59:1442-1444.

18 Iwao K, Nakamori S, Kameyama M, et al. Activation of the beta-catenin gene by interstitial deletions involving exon 3 in primary colorectal carcinomas without adenomatous polyposis coli mutations. Cancer Res 1998;58:1021-1026.
19 Fukuchi T, Sakamoto M, Tsuda H, et al. Beta-catenin mutation in carcinoma of the uterine endometrium. Cancer Res 1998;58:3526-3528.

20 Palacios J, Gamallo C. Mutations in the beta-catenin gene (CTNNB1) in endometrioid ovarian carcinomas. Cancer Res 1998;58:1344-1347.

21 Rashid A, Gao YT, Bhakta S, et al. Beta-catenin mutations in biliary tract cancers: a population-based study in China. Cancer Res 2001;61:3406-3409.

22 Voeller HJ, Truica CI, Gelmann EP. Beta-catenin mutations in human prostate cancer. Cancer Res 1998; 58:2520-2523.

23 Zurawel RH, Chiappa SA, Allen C, et al. Sporadic medulloblastomas contain oncogenic beta-catenin mutations. Cancer Res 1998;58:896-899.

24 Chan EF, Gat U, McNiff JM, et al. A common human skin tumour is caused by activating mutations in betacatenin. Nat Genet 1999;21:410-413.

25 Garcia-Rostan G, Tallini G, Herrero A, et al. Frequent mutation and nuclear localization of beta-catenin in anaplastic thyroid carcinoma. Cancer Res 1999;59: 1811-1815.

26 Tejpar S, Nollet F, Li C, et al. Predominance of beta-catenin mutations and beta-catenin dysregulation in sporadic aggressive fibromatosis (desmoid tumor). Oncogene 1999;18:6615-6620. 